\title{
Mesenchymal Stem Cells: A Promising Therapy for the Acute Respiratory Distress Syndrome
}

\author{
Nayra Cárdenes ${ }^{\mathrm{a}}$ Eder Cáceres $^{\mathrm{a}}$ Militza Romagnoli $^{\mathrm{b}}$ Mauricio Rojas ${ }^{\mathrm{a}, \mathrm{c}, \mathrm{d}}$ \\ ${ }^{a}$ Dorothy P. and Richard P. Simmons Center for Interstitial Lung Disease, University of Pittsburgh School of \\ Medicine, ${ }^{\mathrm{b}}$ Department of Cardiothoracic Surgery, University of Pittsburgh, University of Pittsburgh Medical Center, \\ 'McGowan Institute for Regenerative Medicine, University of Pittsburgh, and d Division of Pulmonary, Allergy and \\ Critical Care Medicine, University of Pittsburgh School of Medicine, Pittsburgh, Pa., USA
}

\section{Key Words}

Acute lung injury · Acute respiratory distress syndrome ·

Alveolar epithelium · Lung injury · Mesenchymal stem cells

\begin{abstract}
Acute respiratory distress syndrome (ARDS) is a pulmonary syndrome with growing prevalence and high mortality and morbidity that increase with age. There is no current therapy able to restore pulmonary function in ARDS patients. Preclinical models of ARDS have demonstrated that intratracheal or systemic administration of mesenchymal stem cells (MSCs) protects the lung against injury. The mechanisms responsible for the protective effects are multiple, including the secretion of multiple paracrine factors capable of modulating the immune response and restoring epithelial and endothelial integrity. Recent studies have demonstrated that MSCs can also control oxidative stress, transfer functional mitochondria to the damaged cells, and control bacterial infection by secretion of antibacterial peptides. These characteristics make MSCs promising candidates for ARDS therapy.

Copyright $\odot 2013$ S. Karger AG, Basel
\end{abstract}

(C) 2013 S. Karger AG, Basel

0025-7931/13/0854-0267\$38.00/0

Fax +4161306 1234

E-Mail karger@karger.com www.karger.com
Accessible online at: www.karger.com/res

\section{Introduction}

The acute respiratory distress syndrome (ARDS) is the cause of $10-15 \%$ intensive care unit (ICU) admissions, and its prevalence increased from 2 to $22 \%$ over a period of 20 years (1988-2008) [1]. Even with the current advances in lung-protective ventilation and fluid management, there still is a high mortality rate that is correlated with age. ARDS is followed by poor survival, diminished quality of life and lung function, high costs associated with long hospitalization and ICU stays, and increased use of health services after hospital discharge. In addition, survivors often have long-term neuromuscular, cognitive, and psychological symptoms with enormous economical and social costs $[2,3]$.

Previous articles in this series: 1. Bouros D, Laurent G: Regenerative medicine and stem cells: Prometheus revisited. Respiration 2013;85:1-2. 2. Kolios G, Moodley Y: Introduction to stem cells and regenerative medicine. Respiration 2013;85:3-10. 3. Ardhanareeswaran K, Mirotsou M: Lung stem and progenitor cells. Respiration 2013;85:89-95. 4. Tzouvelekis A, Ntolios P, Bouros D: Stem cell treatment for chronic lung diseases. Respiration 2013;85:179-192. 
Fig. 1. ARDS produces lung inflammation and edema. E. coli endotoxin was systemically infused to sheep to induce ARDS. The picture shows the lung before (a) and $1 \mathrm{~h}$ after endotoxin administration (b).

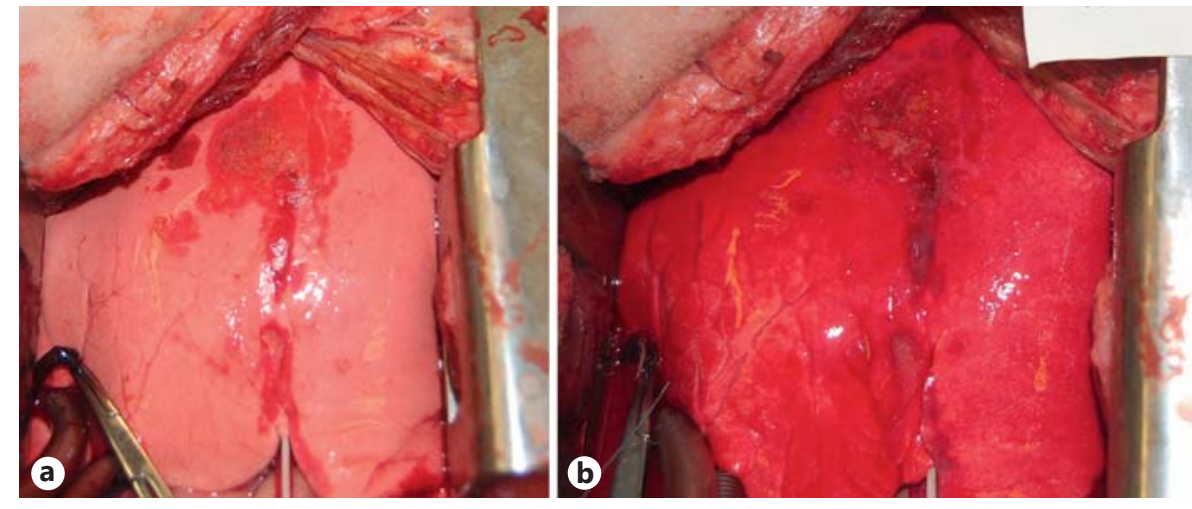

Although existing therapeutic approaches are contributing to the decrease in mortality, they, in many instances, aggravate lung injury. Currently, the only available treatment is limited to supportive care with mechanical ventilation being the key component for improvement in survival outcome. However, since the above treatment often results in further injury to the lungs, there is the imperative need for alternative therapeutic options.

In the last decade, there has been extensive experimentation with multipotent mesenchymal stromal cells or mesenchymal stem cells (MSCs) for the treatment of organ injury. The infusion of exogenous MSCs has been shown to be beneficial in the treatment of induced lung injury in murine animal models [bleomycin, cecal ligation and puncture, ventilation-induced lung injury and endotoxin/lipopolysaccharide (LPS)], as well as for different diseases such as asthma, inflammatory bowel disease, heart disease, diabetes or organ transplant. The curative properties of these cells have been mainly explained by their paracrine effects: the secretion of cytokines and growth factors, which contribute to the modulation of the immune and inflammatory responses. In this review, we describe the most updated definition of ARDS and the relevant biomarkers of the syndrome; we then explain the mechanisms for the therapeutic effects of MSCs in injury, most specifically in ARDS.

\section{The Acute Respiratory Distress Syndrome}

ARDS is a very common clinical entity and a major cause of morbidity ( $5 \%$ of hospitalized, mechanically ventilated patients) [4] and mortality in the critical care setting. Historically, ARDS has been associated with an average hospital mortality of $40 \%$, with worse outcomes in the elderly population. Only in the US, 190,600 new cases of ARDS occur every year $[5,6]$. Moreover, ARDS has a significant impact on long-term disability and adverse psychosocial outcomes in survivors, representing an increase in costs and use of health care services [7].

Incidence rates of morbidity and mortality of ARDS increase with age [6]. Recent studies show an incidence of 306 per 100,000 person-years for people from 75 to 84 years, compared to 16 cases per 100,000 person-years in teenagers between 15 and 19 years. Mortality increases from 24 to $60 \%$ for those older than 85 years compared to 15 - to 19-year-old patients [6]. Furthermore, younger patients had a better rate of recovery than older patients, but neither group returned to the previous level of functionality [8].

In 2011, an updated definition of ARDS was developed with the objective of providing a more accurate and specific definition of the syndrome that addresses previous limitations, such as the lack of explicit criteria to define acuity, sensitivity to ventilation setting adjustments of the arterial oxygenation efficiency $\left(\mathrm{PaFiO}_{2}\right.$ ratio) and the poor reliability of radiographic criteria. In the new Berlin definition [9], diagnostic criteria for ARDS rely on 4 categories: (1) timing: within 1 week of a known clinical insult or new or worsening respiratory symptoms; (2) radiography: bilateral opacities - not fully explained by effusions, lobar/lung collapse or nodule; (3) origin of lung edema: respiratory failure not fully explained by cardiac failure or fluid overload, and (4) oxygenation impairment: subdivided into 3 categories according to the degree of hypoxemia severity (mild, moderate and severe) $[9,10]$. Thus, the Berlin definition eliminated the concept of acute lung injury, which now falls in the category of mild ARDS.

ARDS is the result of a wide variety of disorders with a heterogeneity of hosts, risk factors and other coincident 
processes. It can be associated with different clinical disorders directly affecting the lungs (pneumonia, gastric aspiration or pulmonary contusion) or systemic diseases affecting the lung through the bloodstream (sepsis, severe trauma, acute pancreatitis or transfusions). Regardless of the cause of ARDS, the alveolar epithelium and capillary endothelium are affected, leading to an increase in permeability that allows protein-rich fluid to accumulate in the alveolar space (alveolar edema; fig. 1). Loss of epithelial integrity also disrupts alveolar clearance and production of surfactant [11-13]. In addition to the alveolar damage, there is influx of circulating inflammatory cells and formation of hyaline membranes usually caused by mechanical ventilation [14]. The initial phase of fluid accumulation is followed $72 \mathrm{~h}$ later by a proliferation phase, characterized by an increase in type II alveolar cells, fibroblasts, myofibroblasts and matrix deposition. If the inflammatory process is severe enough, there will be disorganized repair ensuing in fibrosis. Multiple organ failure is the leading cause of death in ARDS patients [15].

The extensive alveolar and endothelial damage causes production of pro-inflammatory cytokines such as TNF- $\alpha$, IL- $1 \beta$ and IL- 6 by alveolar macrophages and endothelial cells. Other factors such as endothelin-1 (EDN1), angiotensin-2 (AGT-II) and phospholipase A2 (PLA2) increase vascular permeability, destroy microvascular architecture and worsen vasoconstriction leading to even more pulmonary hypertension and loss of lung compliance $[16,17]$.

Activation of neutrophils, which release reactive oxygen species (ROS) and proteases, has been recognized in the pathogenesis of ARDS. Elevated levels of ROS and reactive nitrogen species, such as hydrogen peroxide, superoxide anion and nitric oxide, are a distinctive characteristic of the syndrome. These reactive species serve multiple functions, including killing of phagocytosed microorganisms, removal of cell and tissue debris, and induction of signaling events related to inflammation and repair. However, high levels of persistent ROS can also cause tissue necrosis and inflammation contributing to an increase in tissue injury and destruction.

\section{Current Therapies and Support for ARDS}

One reason for the high morbidity and mortality of this disease is the lack of effective treatment available. Currently, the only existing therapy is limited to supportive care. Since the introduction of ventilation with low tidal volumes, there has been a significant decrease in mortality and an increase in the number of days without ventilator use, which often results in further injury to the lungs [18]. Peak end-expiratory pressure is also an essential component of mechanical ventilation which decreases repetitive opening and closing during the respiratory cycle and opens collapsed alveoli decreasing intrapulmonary shunt. Other ventilatory strategies used in ARDS include recruitment maneuvers, prone position and highfrequency ventilation, although the role of these strategies is not well defined and improvement in mortality has not been demonstrated [18].

Another intervention commonly used is the restrictive fluid management and negative fluid balance, which was associated with a better outcome, including an increase in ventilator-free days and shorter ICU stays, but no significant improvement in mortality [19]. There are conflicting data about other therapeutic options, including inhaled NO [2, 20], prostacyclin [21], angiotensin-converting enzyme 2 [22], corticosteroids [23], surfactant replacement [24] and extracorporeal membrane oxygenation [25], which have demonstrated poor effects on outcome and concerning side effects [26].

\section{Biomarkers}

Several plasma molecules have been studied as biomarkers to predict clinical outcome in ARDS. One study evaluated 8 serum biomarkers that were chosen based on prior demonstration of their association with adverse outcomes and included von Willebrand factor, surfactant protein D (SP-D), TNFR-1, IL-6, IL-8, intercellular adhesion molecule, protein $\mathrm{C}$ and plasminogen activator inhibitor-1 [27]. The best performing serum biomarkers were IL-8 (neutrophil chemotactic factor) and SP-D, a product of alveolar type II cells. A combination of clinical predictors and biomarkers was superior to either of them alone for predicting mortality in ARDS [27].

In summary, ARDS is a clinical syndrome with a heterogeneous variety of etiologies and has an important impact on the short- and long-term prognosis of ICU-hospitalized patients. Imbalance of inflammatory response plays a crucial role in ARDS patients, and several cytokines and chemokines have been proposed as biomarkers to predict clinical outcome. Currently, the strongest evidence lies on IL- 8 and SP-D, which have shown to improve performance of clinical predictors [27].

\section{Mesenchymal Stem Cells}

Bone marrow-derived stem cells can be divided in two groups: nonadherent hematopoietic stem cells (HSC) and adherent stem cells that can be further divided into two 
groups: bone marrow-derived MSCs and fibrocytes or fibroblast progenitor cells. MSCs are also able to support hematopoiesis in culture providing extracellular matrix, cytokines and growth factors to the HSC. MSCs are multipotent stromal cells with the potential to go into asymmetrical differentiation by self-renewing and differentiating into a variety of cell lineages [28]. Initially described in a subpopulation of bone marrow-adherent cells, MSCs have now been isolated from a wide variety of tissues, including umbilical cord blood, Wharton's jelly, placenta, adipose tissue and lung. The Mesenchymal and Tissue Stem Cell Committee of the International Society for Cellular Therapy has delineated the minimal criteria that define an MSC. Accurate characterization of MSCs has been a complicated issue since there are no specific cell surface markers. The minimal criteria for establishing the MSC phenotype are: (i) ability to adhere to plastic; (ii) expression of CD44, CD73, CD90 and CD105; (iii) lack of expression of hematopoietic markers CD45, CD34 and CD31, and finally (iv) the possibility to confirm their plasticity by the ability of the cells to differentiate in an in vitro trilineage differentiation assay into adipocytes, osteocytes and chondrocytes [29]. However, their differentiation potential has not been demonstrated in in vivo studies.

Characterization of the contribution of endogenous MSCs during injury has been a complicated issue given that there are no specific chemoattractant or transcription factors that can be used to exclusively inhibit their migration. However, it is well known that MSCs migrate preferentially into the injured organ, as it has been demonstrated in the myocardium [30-33], brain [34, 35], skeletal muscle [36] or kidney [37]. Our group has also proved a preferential migration to the injured lung. To determine whether soluble factors produced by injured lungs can affect stem cell behavior, we developed a simple but effective assay that allows us to measure proliferation and migration influenced by cell suspensions obtained from injured lung. We cocultured mixed cell suspensions prepared from mouse lungs after a single dose of either endotoxin or saline (upper chamber) with green fluorescence protein (GFP) + MSCs (bottom chamber). Lung cells were separated from MSCs by a $3-\mu \mathrm{m}$ pore membrane, limiting the crossing from one chamber to the other to soluble factors. Cocultures were maintained for 5 days, after which the upper chamber and the membrane were examined by fluorescence microscopy. In this setting, we were able to demonstrate a marked migration of the GFP+ MSCs towards the cell suspensions from the endotoxin-injured but not the control lung cells. We later confirmed these results in a murine model of bleomycin- induced fibrosis, in which infused MSCs were only detected in animals treated with bleomycin. In control animals that received PBS, MSCs were not detected in the lung [38].

MSCs are also known to be 'immunoprivileged', as they do not easily trigger host cell responses. It is thought that T4 lymphocytes are not able to recognize them as foreign due to their low expression of the major histocompatibility complex proteins and of T-cell costimulatory proteins. All of this may play an important role in tissue regeneration and repair. MSCs have been shown to induce T-cell apoptosis via the FAS-L-dependent FAS pathway; apoptotic T cells trigger macrophages to produce high levels of TGF- $\beta$, which upregulates CD4+, CD25+, FoxP3+ regulatory T cells and immune tolerance [39]. Naive T cells were also induced to differentiate into regulatory $\mathrm{T}$ cells (FoxP3+) by MSC TGF- $\beta$ secretion (fig. 2). Thanks to this unique characteristic, we were able to break the immune barrier by using MSCs isolated from different mouse strains to prevent airway occlusion on the heterotopic trachea transplantation model. Two to 4 tracheas obtained from $\mathrm{BALB} / \mathrm{c}$ mice were placed in the back of a C57BL/6 mouse. Intravenous infusion of bone marrow-derived MSCs obtained from $\mathrm{CH} 3, \mathrm{BALB} / \mathrm{c}$ and C57BL/6 all showed the same level of protection, thus confirming the ability of bone marrow-derived MSCs to escape the surveillance of the immune system [40].

Several studies have demonstrated the potential therapeutic effect of MSCs in different diseases: myocardial infarction [41-44], pulmonary fibrosis [45, 46], pulmonary hypertension [47-50], emphysema [51-53], diabetes [54], sepsis [55, 56], asthma [57], hepatic failure [58], acute renal failure [59] and ARDS [5, 38, 60-64]. In all preclinical studies, infused MSCs expanded in vitro were used.

To indirectly demonstrate the importance of endogenous MSCs, we decided to correlate the decrease in MSC function with the general decline associated with age. We first demonstrated in different models that there is an increase in the susceptibility to lung injury in old mice. In order to study how aging of MSCs affects their ability to repair the tissue, we and others have characterized MSCs morphologically and functionally in different age groups. It is now known that MSCs from elderly people have different morphology, increased production of ROS and oxidative damage [65], DNA methylation changes affecting cell differentiation [66], slower proliferation in culture $[67,68]$, shorter telomeres $[68]$ and a large proportion of them stain positive for senescence-associated $\beta$-galactosidase [69]. We have defined a senescence-related increase in mobilization of fibroblast progenitor cells or 


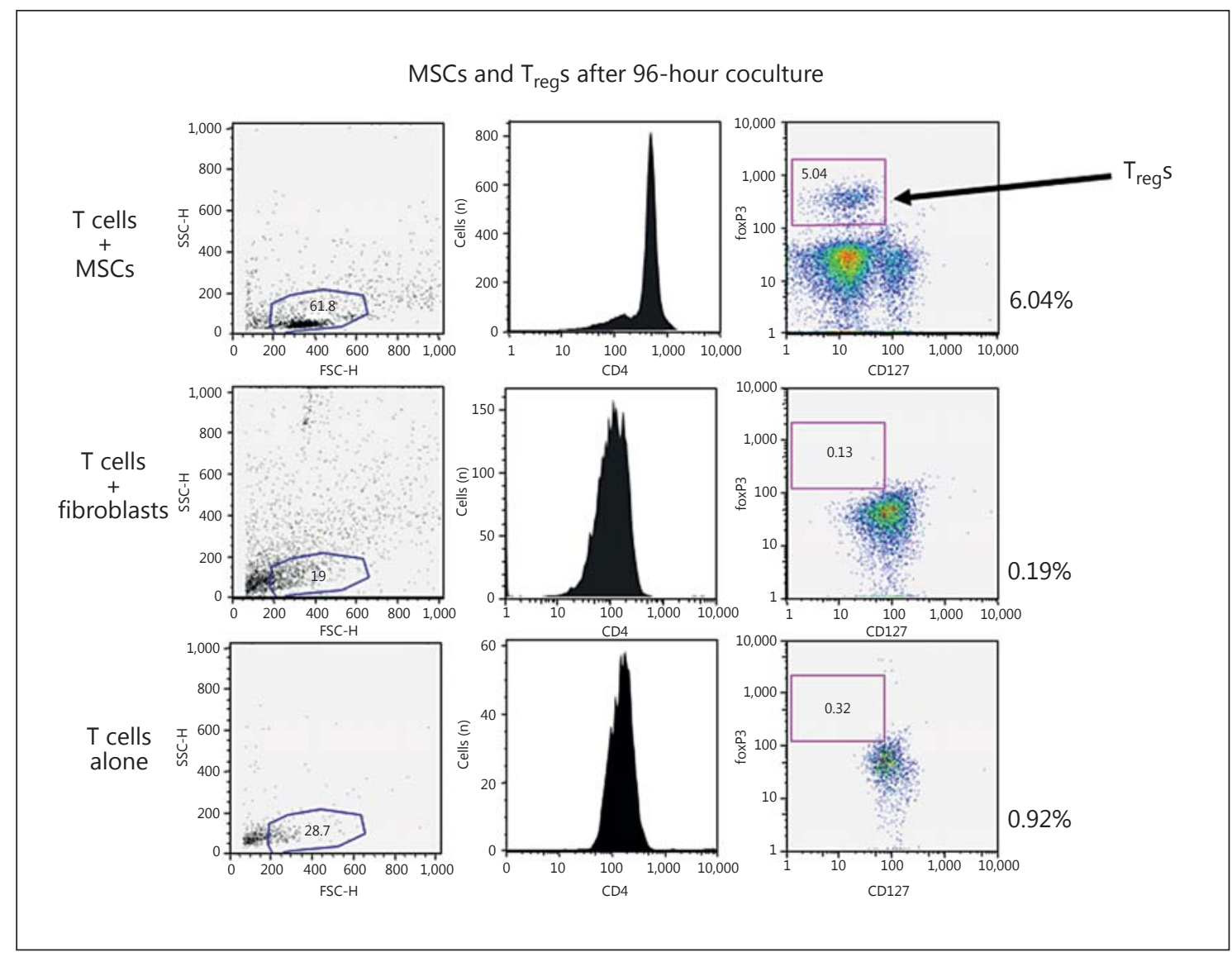

Fig. 2. $T$ cells differentiate into T-regulatory cells $\left(\mathrm{T}_{\text {reg }} \mathrm{s}\right)$ when cocultured with MSCs. $\mathrm{T}$ cells were cocultured with MSCs, fibroblasts or alone for $96 \mathrm{~h}$. Only when T cells were cultured in the presence of MSCs, they were Tcell marker CD4 and regulatory marker FoxP3 positive.

fibrocytes in aging mice associated with a simultaneous decrease in MSC migration, which is linked to a poor response to high serum levels of Cxcl12. Alt et al. [70] compared adipose tissue derived-MSCs from 3 age groups (young, adult and old) of healthy volunteers. They found that aging not only reduces the proliferative capacity and differentiation potential of MSCs, it also affects the expression of DNA break repair genes. In a recent study based on human MSCs from very young (0-6 years), adult (25-50 years) and old donors ( $>60$ years), MSCs from the very young donors maintained their fibroblastic-like morphology up to higher passages and proliferated in a greater number than adult and old donors. The osteogenic and neurogenic differentiation potential also decreased with age [71]. Additionally, MSCs from old mice are characterized by a quiescent state with low metabolic activity and are primarily in the G0 phase of the cell cycle. This quiescent state is maintained by both extrinsic and intrinsic mechanisms and has been postulated to be a way of preserving their long-term proliferative potential and genomic integrity. The problem that arises is that quiescent MSCs escape DNA damage checkpoints and several repair pathways that are cell cycle dependent, which results in the accumulation of DNA damage during aging, ultimately leading to apoptosis of the stem cell and therefore MSC depletion [72-78]. The critical effect of accumulation of DNA damage in aging MSCs has been recently demonstrated using a progeroid mouse model with defects in DNA repair due to a deficiency in the excision repair cross-complementation group (Ercc1) protein [79-83]. Unpublished data by our group have demonstrated in the parabiotic setting that an Ercc1-deficient mouse benefits from a longer life span when surgically joined to a wild-type mouse, compared to when 2 Ercc1 mice are connected. This observation correlates with the recently published work by Lavasani et al. [84] who dem- 
onstrated that only the transfer of wild-type muscle-derived MSCs into Ercc1-deficient mice is sufficient to halt premature aging and to increase life span. In another insightful study, an old mouse with declining organ stem cell capacity underwent rejuvenation of aged muscle and liver progenitor cells when surgically joined to a young mouse [85]. In a rat model of cardiomyopathy, human MSCs from aged donors did not perform as well as the ones from young donors [86]. MSCs isolated from old donors failed to differentiate in vitro into bone, cartilage and neuroectodermal cells with an increase in their ability to transform in adiposites [87]. The superiority of very young MSCs can be explained by several aspects of their biology [88]. Preservation of their undifferentiated stage is a critical step to retain their ability to repair, as it is known that after cells differentiate they express MHC classes I and II allowing them to be recognized and eliminated by immune system.

MSCs secrete multiple factors, such as growth factors, factors regulating endothelial and epithelial permeability and anti-inflammatory cytokines, which can modulate the immune response and facilitate repair and regeneration. Cell-to-cell contact seems to be a key mechanism by which MSCs modulate immune effector cells such as macrophages and T cells. TNF- $\alpha$-activated MSCs appear to stimulate macrophages to produce more IL-10 through the release of prostaglandin $\mathrm{E}_{2}$ (PGE-2), which acts on EP2 and EP4 macrophage receptors [55]. MSCs stimulated by cytokines such as IFN- $\gamma$, TNF- $\alpha$ and IL- $1 \beta$ have shown antibacterial properties by inducing activity of indoleamine 2,3-dioxygenase, a tryptophan-catabolizing enzyme, leading to tryptophan starvation in bacteria, protozoa and viruses [89]. Increased expression of other antimicrobial peptides is also associated with protective effects of MSCs. Human cathelicidin, hCAP-18/LL-37, exerts its microbicidal activity through the disruption of the bacterial membrane, chemotaxis and chemokine induction $[90,91]$. In addition to the secreted molecules, transference of mitochondria from MSCs to type II pneumocytes through nanotubules has been demonstrated to occur in mouse lungs improving surfactant production and preventing apoptosis [92].

In summary, MSCs broadly suppress T-cell activation and proliferation and induce differentiation of T-regulatory cells via soluble and cell contact-dependent mediators. These mediators may act directly upon $\mathrm{T}$ cells or indirectly via modulation of antigen-presenting cells and other accessory cells. These properties give MSCs an important therapeutic potential for diseases in which acute and chronic inflammatory responses are implicated.
The fact that MSCs have immunomodulatory properties and low immunogenicity, together with their ability to respond to injury by secreting multiple soluble factors that can promote organ repair, makes them a promising therapy for different inflammatory diseases. In general, the development of cell therapies has taken an important role as an alternative treatment for major diseases mostly because they are unique in their ability to control inflammation as well as protect the endothelium and the epithelium.

\section{MSCs in ARDS Therapy}

The ability of MSCs to create a tolerogenic niche by direct interaction with immune cells and by secretion of regulatory molecules makes them attractive therapeutic candidates in the regulation of acute inflammatory responses to infection and injury in the lung. Extensive research has been done regarding the therapeutic effects of MSCs in ARDS in in vivo and ex vivo models with promising results. Several studies, including publications from our group, have demonstrated compelling benefits from the administration of MSCs in animal models of ARDS (table 1). Administration of bacterial LPS (endotoxin/ LPS) to mice produces pathophysiologic changes similar to those seen in ARDS in humans. In a murine model of ARDS, exogenous infusion of MSCs prevents the systemic inflammatory response to endotoxin and attenuates lung injury [38, 46, 93] (fig. 3).

The mechanisms by which MSCs contribute to lung injury repair have been explored at different levels (fig. 4). In order for the cells to have any beneficial effect in the wounded area, they need to interact with the injured niche. Fluorescence labeling of MSCs has made it possible to observe the homing of these cells to the injured lungs $[64,94]$. A recent study in mice has shown that $60 \%$ of radioactively labeled MSCs administered to mice home to the lung in the first $24 \mathrm{~h}$ [95]. However, evidence has suggested that the migration of MSC is favored by tissue injury [96]. As mentioned above, in the murine model of ARDS, exogenous infusion of MSCs prevents the inflammatory response to endotoxin. The inflammatory state is characteristic of ARDS. In a series of unpublished data, human MSCs were cultured with $0.5 \%$ serum from ARDS patients in order to test whether the immunomodulatory capacity of human MSCs was activated once the cells were in contact with the proinflammatory environment characteristic of ARDS. The results showed an increased level of the anti-inflammatory cytokine IL-10. In contrast, there was a decrease in inflammatory cytokine levels: IL-6, IL-1 $\beta$ and IL-8. 
Table 1. Preclinical models of MSCs in ARDS

\begin{tabular}{|c|c|c|}
\hline Study & MSC route & Clinical outcome \\
\hline \multicolumn{3}{|c|}{ Murine bleomycin-induced ARDS } \\
\hline $\begin{array}{l}\text { Ortiz et al. [108] } \\
(2007)\end{array}$ & $\begin{array}{l}\text { i.v. (immediately } \\
\text { after injury) }\end{array}$ & $\begin{array}{l}\text { Decrease in BAL protein } \\
\text { Secretion of IL- } 1 \text { receptor antagonist } \\
\text { MSC administration more effective than recombinant IL1RN in inhibiting } \\
\text { immune cell trafficking and cytokine expression }\end{array}$ \\
\hline $\begin{array}{l}\text { Zhao et al. [109] } \\
(2008)\end{array}$ & i.v. (12 $\mathrm{h}$ after injury) & $\begin{array}{l}\text { Decreased content of hydroxyproline, laminin and hyaluronan in BAL, } \\
\text { ARDS markers, fibrosis, and proinflammatory cytokines }\end{array}$ \\
\hline $\begin{array}{l}\text { Lee et al. [110] } \\
(2010)\end{array}$ & $\begin{array}{l}\text { i.v. ( } 4 \text { days after } \\
\text { injury) }\end{array}$ & $\begin{array}{l}\text { Histologic decrease in lung injury } \\
\text { Decrease in excess lung water } \\
\text { Restored alveolar fluid clearance }\end{array}$ \\
\hline
\end{tabular}

Murine cecal ligation and puncture-induced ARDS

\begin{tabular}{lll}
$\begin{array}{l}\text { Gonzalez-Rey et al. } \\
{[111] \text { (2009) }}\end{array}$ & $\begin{array}{l}\text { i.p. (7 days } \\
\text { after injury) }\end{array}$ & $\begin{array}{l}\text { Ameliorated clinical and histopathological severity of colitis, with no weight loss, } \\
\text { diarrhea and inflammation, and increasing survival } \\
\text { Downregulation of the Th1-driven inflammatory responses }\end{array}$ \\
$\begin{array}{l}\text { Nemeth et al. [55] } \\
(2009)\end{array}$ & $\begin{array}{l}\text { i.v. (24 h before } \\
\text { and } 1 \text { h after injury) }\end{array}$ & $\begin{array}{l}\text { Reprogramming of macrophages by the release of prostaglandin } \mathrm{E}_{2} \\
\text { Improved survival and organ dysfunction }\end{array}$ \\
\hline $\begin{array}{l}\text { Mei et al. [56] } \\
(2010)\end{array}$ & $\begin{array}{l}\text { i.v. (6 and } 24 \mathrm{~h} \\
\text { after injury) }\end{array}$ & $\begin{array}{l}\text { Reduced mortality } \\
\text { Reduced systemic and pulmonary cytokines, and modification of transcriptional } \\
\text { inflammatory gene activity } \\
\text { Significantly greater bacterial clearance with enhanced phagocytotic activity of } \\
\text { the host immune cells }\end{array}$ \\
\end{tabular}

Murine in vivo and ex vivo endotoxin/Escherichia coli-induced ARDS

Xu et al. [38] i.v. (1 and $24 \mathrm{~h} \quad$ Migration of stem cells to injured lung cells and suppressed proinflammatory

(2007) after injury)

cytokine production by lung cells

Low cell engraftment

Production of stem cell chemoattractants by the lungs and to humoral and physical interactions between stem cells and lung cells

\begin{tabular}{|c|c|c|}
\hline $\begin{array}{l}\text { Gupta et al. [93] } \\
(2007)\end{array}$ & $\begin{array}{l}\text { i.t. ( } 4 \text { and } 24 \mathrm{~h} \\
\text { after injury) }\end{array}$ & $\begin{array}{l}\text { Downregulation of proinflammatory responses to endotoxin } \\
\text { (reducing TNF- } \alpha \text { and MIP-2 in BAL and plasma) } \\
\text { Increase in the anti-inflammatory cytokine IL-10 } \\
\text { Decrease in the severity of endotoxin-induced acute lung injury and improved } \\
\text { survival }\end{array}$ \\
\hline $\begin{array}{l}\text { Lee et al. [107] } \\
\text { (2009) }\end{array}$ & $\begin{array}{l}\text { i.t. ( } 1 \text { and } 24 \mathrm{~h} \\
\text { after injury) }\end{array}$ & $\begin{array}{l}\text { Reduced extravascular lung water, improved lung endothelial barrier } \\
\text { permeability and restored alveolar fluid clearance } \\
\text { Alveolar epithelial fluid transport through the secretion of KGF }\end{array}$ \\
\hline $\begin{array}{l}\text { Iyer et al. [112] } \\
(2010)\end{array}$ & i.p. (after injury) & $\begin{array}{l}\text { MSC infusion leads to a more reducing Cys and GSH redox state } \\
\text { MSC have antioxidant effects in vivo, as part of their effects in lung injury }\end{array}$ \\
\hline $\begin{array}{l}\text { Danchuk et al. [113] } \\
\text { (2011) }\end{array}$ & $\begin{array}{l}\text { i.v., i.p. or oropharyngeal } \\
\text { aspiration }\end{array}$ & Secretion of TSG-6 resulting in reduced neutrophil acitivation and migration \\
\hline
\end{tabular}

Gene plus cell therapy (murine endotoxin/E. coli-induced ARDS)

Mei et al. [64] i.v. (30 min after injury; Reduced acute pulmonary inflammation

(2007) \pm ANGPT-1) Near complete reversal of increased lung permeability with gene transfection

$\mathrm{Xu}$ et al. [106] i.v. (2 h after injury; Further histologic decrease in lung injury with gene transfection vs. MSCs alone

(2008) $\quad \pm$ ANGPT-1) $\quad$ Further decrease excess lung water, proinflammatory cytokines and BAL

protein vs. MSC alone 
Fig. 3. Administration of MSCs in mice decreases endotoxin-induced lung injury. Injured lungs treated with MSCs are devoid of cellular congestion and increase in cellularity, predominantly neutrophils, in contrast with untreated lungs or those treated with fibroblasts [38].
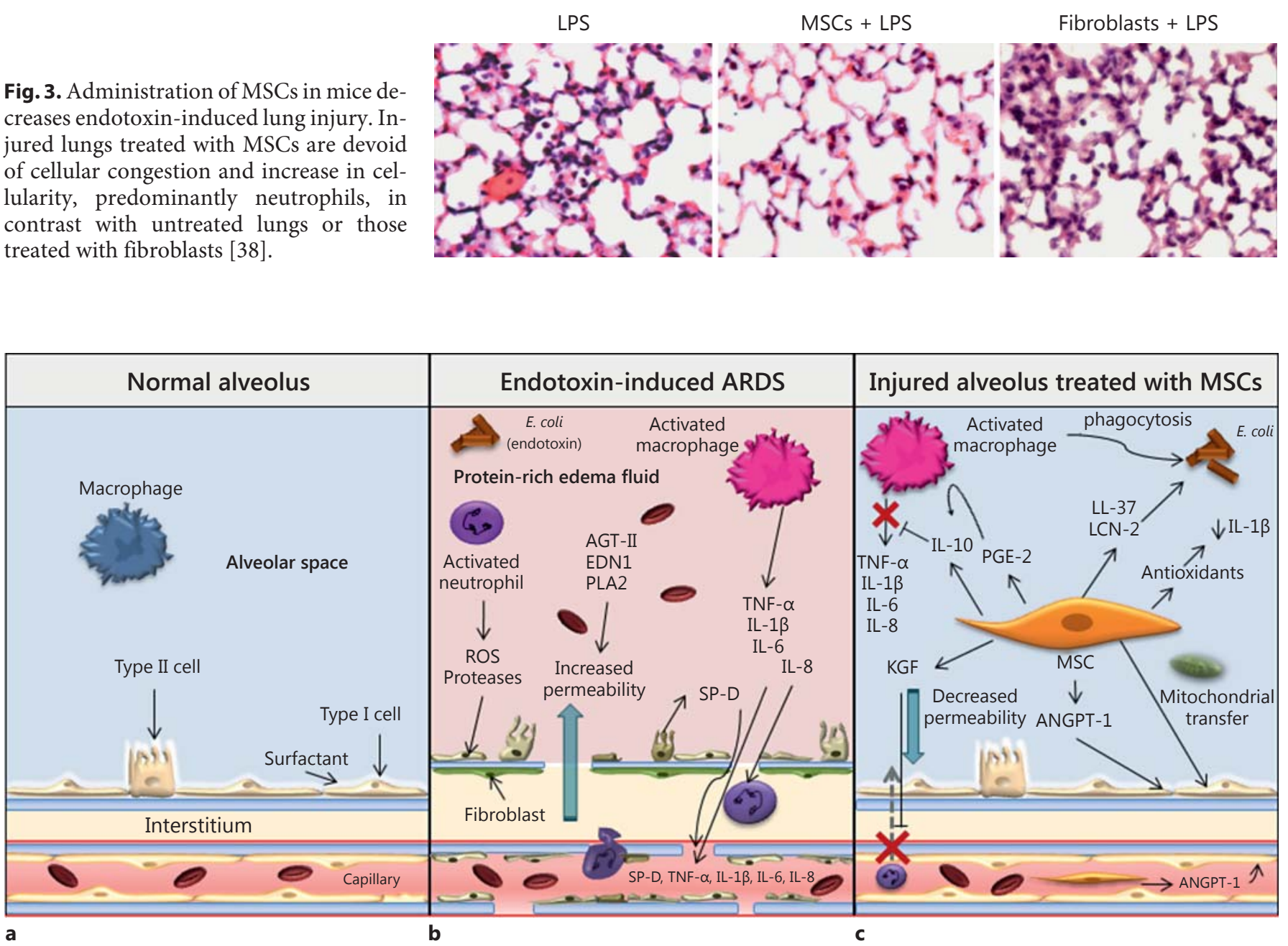

Fig. 4. Comparison of the mechanisms of action in normal, injured and MSC-treated alveoli. In the normal alveolus (a), there is normal fluid movement from the vascular to the interstitial space, with normal architecture of the epithelium and the endothelium, and production of surfactant. b Injured alveolus. Bacterial toxin activates macrophages, which secrete a battery of proinflammatory cytokines that are leaked into the bloodstream. Among these cytokines, IL- 8 is responsible for the recruitment of neutrophils into the injured alveolus. ROS and proteases released by the activated neutrophils damage the alveolar epithelium and endothelium, and, as a result, there is an increase in the permeability and in the

There is strong evidence that infusion of MSCs, after endotoxin-induced ARDS, results in significantly lower levels of inflammatory cytokines, e.g. TNF- $\alpha$, MIP-2, IFN- $\gamma$, IL-1 $\beta$ [97], MIP-1 $\alpha$, IL-6 and the murine homolog of IL-8, keratinocyte-derived cytokine, both in plasma and bronchoalveolar lavage (BAL) $[38,46,93]$. In vitro Transwell coculture of stimulated macrophages and MSCs also showed lower levels of TNF- $\alpha$ but increased influx of protein-rich edema. Another characteristic in ARDS is the increased levels of SP-D by type II cells. c Representation of a restored alveolus that has been treated with MSCs after bacterial injury. MSCs themselves produce and stimulate the production of IL-10 by macrophages, which attenuates their inflammatory cascade. The secretion of ANGPT-1 and the transfer of mitochondria to the epithelial cells by MSCs together with the production of KGF contribute to restoring the epithelium and endothelium. This results in improved alveolar fluid clearance and decreased permeability. MSCs are also responsible for the secretion of antibacterial peptides that participate in the clearance of bacteria.

levels of the anti-inflammatory cytokine IL-10 upon endotoxin stimulation [93]. IL-10 has been shown to inhibit neutrophil migration into tissues, therefore reducing the damage [98]. According to our observations and those by Gupta et al. [93], the protective effect seen with MSC therapy did not require a high level of engraftment in the lung. 
As mentioned before, a consequence of ARDS is the increase in ROS. There is evidence suggesting that MSCs may also be enriched for the presence of antioxidant-protective genes. A number of studies have also investigated the effects on ROS production and antioxidant levels in MSCs. Takahata et al. [99] reported that stimulation of MSCs with adrenaline led to an increase in cellular GSH levels via a process involving Nrf-2-mediated activation of the CySS/glutamate pathway. MSCs cultured at $32^{\circ} \mathrm{C}$ expressed lower ROS levels and demonstrated decreased NO levels, and a decrease in markers of oxidative stress such as malondialdehyde and protein carbonyl content. Interestingly, the decrease in oxidant stress was associated with a decrease in apoptosis. Studies have suggested a reciprocal interaction between MSCs and the extracellular redox state. We have found that MSCs exposed to an oxidized extracellular Cys/CySS in vitro results in a twofold upregulation in cellular ROS production. Because the Cys/CySS redox state is oxidized in endotoxin-induced ARDS, understanding the effects of Cys/CySS redox on MSC function and anti-inflammatory effects is paramount. Results from our group suggest that production of the IL-1 antagonist is decreased in endotoxinstimulated cells that are exposed to an oxidized Cys/CySS compared to the physiological redox state [100]. Additionally, studies in a mouse model of LPS-induced ARDS infused with MSCs result in an improvement in Cys and GSH homeostasis. In summary, in addition to modulating the systemic inflammatory environment, infusion of MSCs alters the systemic redox environment to a less oxidizing value; however, the mechanistic basis for these changes is still unclear. These findings suggest that therapies to preserve oxidation of Cys/CySS during ARDS may improve their protective effect.

Interestingly, since 2006 there have been a number of studies that report the transfer of mitochondria, which are a major source of ROS, from MSCs to the epithelium. Spees et al. [101] showed that human MSCs could rescue mitochondrial function in epithelial cells containing nonfunctional mitochondria. This was confirmed recently in a mouse model of LPS-induced ARDS, where mitochondria from instilled human MSCs were observed optically to be transferred to the mouse lung epithelium. In this study, lung protection was abrogated when MSCs with defective mitochondria were used, and this transfer seemed to be connexin 43 dependent [102]. Whether the beneficial paracrine effects from MSCs are dependent on the attachment of the cells to the lung epithelium, as well as whether the mitochondrial transfer is complementary to the secretion of the paracrine factors, is still not clear.
Because ARDS commonly has a septic component [6], the question was raised on whether the potent anti-inflammatory properties of MSCs would affect host response against infections. Experimental mouse models of sepsis have shown that MSCs not only are able to reduce bacterial counts by an order of magnitude, but also increase the expression of genes involved in phagocytosis $[55,56]$. Most strikingly, it has been demonstrated that MSCs exert antibacterial effects through the secretion of antibacterial peptides human cathelicidin (LL-37) and lipocalin-2 (LCN-2) [90, 103]. Conditioned media from stimulated MSCs were found to contain high levels of antibacterial peptides and to inhibit bacterial growth; however, when incubated with a blocking antibody against these peptides, the media lost their antibacterial properties. This effect was also abrogated in vivo, when MSCs were administered together with the antibodies.

One of the hallmarks of ARDS is the accumulation of alveolar edema due to impaired alveolar fluid clearance and increased microvascular permeability of the lung endothelium, and it is used as a prognostic tool for morbidity and mortality $[104,105]$. The amount of protein or albumin in BAL is commonly used as an indicator of endothelial permeability. In the murine endotoxin model of ARDS, albumin concentration increased in BAL fluid 3 days after the insult, an effect that was diminished with the intravenous administration of MSCs [64]. Additionally, it has been shown by others and us that lung edema is significantly abrogated in animals treated with MSCs or fibrocyte-depleted bone marrow $[38,93]$.

MSCs are also able to restore the alveolar epithelium and endothelial integrity and permeability. Using an innovative cell-based and gene-based approach, $\mathrm{Xu}$ et al. [106] reported that the protective effect of MSCs in the LPS-injured mouse lung is greatly potentiated by infusion of MSCs overexpressing angiopoietin 1 (ANGPT-1), a vasculoprotective gene. MSC infusion significantly decreased airspace neutrophil and pulmonary vascular endothelial permeability after LPS administration. One mechanism that has been proposed for the explanation of the improvement in epithelial integrity is the effect of keratinocyte growth factor (KGF), a soluble factor that participates in the regulation of alveolar fluid transport and is secreted by MSCs. When KGF expression was inhibited on MSCs by iRNA treatment against KGF, the beneficial effects of MSCs or their conditioned medium was diminished by $80 \%$ [107]. These results support the observation that, in the case of ARDS, the effect of MSCs is not only by modulating inflammation but also by protecting local endothelium and epithelium. 


\section{Conclusion}

ARDS is a common disorder with high morbidity and mortality globally. Since ARDS was initially defined, advances in research have contributed to the understanding of the mechanisms underlying the biological causes of the disease, as well as to the short- and long-term effects on outcomes of the current therapies and the development of new ones.

From their original description in the bone marrow, 40 years ago, to the current applications in multiple acute and chronic diseases, MSCs have been a bench-to-bedside paradigm. Because of their ability to respond to injury by different mechanisms, i.e. secretion of multiple paracrine factors (such as growth factors and anti-inflammatory cytokines), the ability to control oxidative damage, protection of the endothelium and the epithelium, transfer of functional mitochondria, and more recently, through the secretion of antimicrobial peptides, MSCs have become an exciting candidate for novel promising therapies for patients with ARDS.

\section{Acknowledgments}

We want to thank Maria Kapetanaki and Theresa Heinrich for critical comments and editing.

\section{References}

1 Rincon F, et al: Impact of acute lung injury and acute respiratory distress syndrome after traumatic brain injury in the United States. Neurosurgery 2012;71:795-803.

2 Angus DC, et al: Healthcare costs and longterm outcomes after acute respiratory distress syndrome: a phase III trial of inhaled nitric oxide. Crit Care Med 2006;34:2883-2890.

3 Walkey AJ, et al: Acute respiratory distress syndrome: epidemiology and management approaches. Clin Epidemiol 2012;4:159-169.

4 Serpa Neto A, et al: Association between use of lung-protective ventilation with lower tidal volumes and clinical outcomes among patients without acute respiratory distress syndrome: a meta-analysis. JAMA 2012;308: 1651-1659.

5 Hayes M, et al: Clinical review: stem cell therapies for acute lung injury/acute respiratory distress syndrome - hope or hype? Crit Care 2012;16:205.

6 Rubenfeld GD, et al: Incidence and outcomes of acute lung injury. N Engl J Med 2005;353: 1685-1693.

7 Davidson TA, et al: The effect of acute respiratory distress syndrome on long-term survival. Am J Respir Crit Care Med 1999;160:18381842.

8 Herridge MS, et al: Functional disability 5 years after acute respiratory distress syndrome. N Engl J Med 2011;364:1293-1304.

-9 Ranieri VM, et al: Acute respiratory distress syndrome: the Berlin definition. JAMA 2012; 307:2526-2533.

10 Ferguson ND, et al: The Berlin definition of ARDS: an expanded rationale, justification, and supplementary material. Intensive Care Med 2012;38:1573-1582.

11 Berger G, et al: Sepsis impairs alveolar epithelial function by downregulating $\mathrm{Na}-\mathrm{K}$ ATPase pump. Am J Physiol Lung Cell Mol Physiol 2011;301:L23-L30.
12 Vadasz I, Weiss CH, Sznajder JI: Ubiquitination and proteolysis in acute lung injury. Chest 2012;141:763-771.

13 Piantadosi CA, Schwartz DA: The acute respiratory distress syndrome. Ann Intern Med 2004;141:460-470.

14 Ware LB, Matthay MA: Clinical practice. Acute pulmonary edema. N Engl J Med 2005; 353:2788-2796

15 Erickson SE, et al: Recent trends in acute lung injury mortality: 1996-2005. Crit Care Med 2009;37:1574-1579.

16 Proudfoot AG, Hind M, Griffiths MJ: Biomarkers of acute lung injury: worth their salt? BMC Med 2011;9:132.

17 Tzouvelekis A, Pneumatikos I, Bouros D: Serum biomarkers in acute respiratory distress syndrome an ailing prognosticator. Respir Res 2005;6:62.

18 Ventilation with lower tidal volumes as compared with traditional tidal volumes for acute lung injury and the acute respiratory distress syndrome. The Acute Respiratory Distress Syndrome Network. N Engl J Med 2000;342: 1301-1308.

19 Wiedemann HP, et al: Comparison of two fluid-management strategies in acute lung injury. N Engl J Med 2006;354:2564-2575.

20 Numata $M$, et al: Inhibition of inducible nitric oxide synthase prevents LPS-induced acute lung injury in dogs. J Immunol 1998; 160: 3031-3037.

21 Camamo JM, McCoy RH, Erstad BL: Retrospective evaluation of inhaled prostaglandins in patients with acute respiratory distress syndrome. Pharmacotherapy 2005;25:184190.

22 Treml B, et al: Recombinant angiotensin-converting enzyme 2 improves pulmonary blood flow and oxygenation in lipopolysaccharideinduced lung injury in piglets. Crit Care Med 2010;38:596-601.
23 Khilnani GC, Hadda V: Corticosteroids and ARDS: a review of treatment and prevention evidence. Lung India 2011;28:114-119.

24 Lewis JF, Veldhuizen RA: The future of surfactant therapy during ALI/ARDS. Semin Respir Crit Care Med 2006;27:377-388.

25 Combes A, et al: Extracorporeal membrane oxygenation for respiratory failure in adults. Curr Opin Crit Care 2012;18:99-104.

26 Barbas CS, et al: Goal-oriented respiratory management for critically ill patients with acute respiratory distress syndrome. Crit Care Res Pract 2012;2012:952168.

27 Ware LB, et al: Prognostic and pathogenetic value of combining clinical and biochemical indices in patients with acute lung injury. Chest 2010;137:288-296.

28 McCulloch EA, Till JE: Perspectives on the properties of stem cells. Nat Med 2005;11: 1026-1028.

29 Dominici M, et al: Minimal criteria for defining multipotent mesenchymal stromal cells. The International Society for Cellular Therapy position statement. Cytotherapy 2006;8: 315-317.

$30 \mathrm{Wu}$ Y, Zhao RC: The role of chemokines in mesenchymal stem cell homing to myocardium. Stem Cell Rev 2012;8:243-250.

31 Shim W, et al: G-CSF for stem cell therapy in acute myocardial infarction: friend or foe? Cardiovasc Res 2011;89:20-30.

32 Bartsch K, et al: Mesenchymal stem cells remain host-derived independent of the source of the stem-cell graft and conditioning regimen used. Transplantation 2009;87:217-221.

33 Schenk S, et al: Monocyte chemotactic protein-3 is a myocardial mesenchymal stem cell homing factor. Stem Cells 2007;25:245-251. 
34 Ji JF, et al: Interactions of chemokines and chemokine receptors mediate the migration of mesenchymal stem cells to the impaired site in the brain after hypoglossal nerve injury. Stem Cells 2004;22:415-427.

-35 Munoz-Elias G, et al: Adult bone marrow stromal cells in the embryonic brain: engraftment, migration, differentiation, and longterm survival. J Neurosci 2004;24:4585-4595.

-36 De Bari C, et al: Skeletal muscle repair by adult human mesenchymal stem cells from synovial membrane. J Cell Biol 2003;160:909-918.

37 Morigi M, et al: Mesenchymal stem cells are renotropic, helping to repair the kidney and improve function in acute renal failure. J Am Soc Nephrol 2004;15:1794-1804.

38 Xu J, et al: Prevention of endotoxin-induced systemic response by bone marrow-derived mesenchymal stem cells in mice. Am J Physiol Lung Cell Mol Physiol 2007;293:L131L141.

-39 Akiyama K, et al: Mesenchymal-stem-cell-induced immunoregulation involves FAS-ligand-/FAS-mediated T cell apoptosis. Cell Stem Cell 2012;10:544-555.

40 Grove DA, et al: Attenuation of early airway obstruction by mesenchymal stem cells in a murine model of heterotopic tracheal transplantation. J Heart Lung Transplant 2011;30: 341-350.

-41 Li TS, et al: Regeneration of infarcted myocardium by intramyocardial implantation of ex vivo transforming growth factor-beta-preprogrammed bone marrow stem cells. Circulation 2005;111:2438-2445.

42 Miyahara Y, et al: Monolayered mesenchymal stem cells repair scarred myocardium after myocardial infarction. Nat Med 2006;12:459465.

43 Iso Y, et al: Multipotent human stromal cells improve cardiac function after myocardial infarction in mice without long-term engraftment. Biochem Biophys Res Commun 2007; 354:700-706.

44 Lee RH, et al: Intravenous hMSCs improve myocardial infarction in mice because cells embolized in lung are activated to secrete the anti-inflammatory protein TSG-6. Cell Stem Cell 2009;5:54-63.

45 Ortiz LA, et al: Mesenchymal stem cell engraftment in lung is enhanced in response to bleomycin exposure and ameliorates its fibrotic effects. Proc Natl Acad Sci USA 2003; 100:8407-8411.

46 Rojas M, et al: Bone marrow-derived mesenchymal stem cells in repair of the injured lung. Am J Respir Cell Mol Biol 2005;33:145-152.

47 Luan Y, et al: Mesenchymal stem cell prevention of vascular remodeling in high flow-induced pulmonary hypertension through a paracrine mechanism. Int Immunopharmacol 2012;14:432-437.

-48 Kim KC, et al: Changes of gene expression after bone marrow cell transfusion in rats with monocrotaline-induced pulmonary hypertension. J Korean Med Sci 2012;27:605-613.
49 Stewart DJ, Mei SH: Cell-based therapies for lung vascular diseases: lessons for the future. Proc Am Thorac Soc 2011;8:535-540.

50 Luan Y, et al: Implantation of mesenchymal stem cells improves right ventricular impairments caused by experimental pulmonary hypertension. Am J Med Sci 2012;343:402-406.

51 de Faria CA, et al: Experimental basis and new insights for cell therapy in chronic obstructive pulmonary disease. Stem Cell Rev 2012;8: 1236-1244.

52 Guan XJ, et al: Mesenchymal stem cells protect cigarette smoke-damaged lung and pulmonary function partly via VEGF-VEGF receptors. J Cell Biochem 2013;114:323-335.

53 Huh JW, et al: Bone marrow cells repair cigarette smoke-induced emphysema in rats. Am J Physiol Lung Cell Mol Physiol 2011; 301:L255-L266.

54 Lee RH, et al: Multipotent stromal cells from human marrow home to and promote repair of pancreatic islets and renal glomeruli in diabetic NOD/scid mice. Proc Natl Acad Sci USA 2006; 103:17438-17443.

55 Nemeth K, et al: Bone marrow stromal cells attenuate sepsis via prostaglandin E(2)-dependent reprogramming of host macrophages to increase their interleukin-10 production. Nat Med 2009;15:42-49.

56 Mei SH, et al: Mesenchymal stem cells reduce inflammation while enhancing bacterial clearance and improving survival in sepsis. Am J Respir Crit Care Med 2010;182:10471057.

57 Firinci F, et al: Mesenchymal stem cells ameliorate the histopathological changes in a murine model of chronic asthma. Int Immunopharmacol 2011;11:1120-1126.

58 Parekkadan B, et al: Mesenchymal stem cellderived molecules reverse fulminant hepatic failure. PLoS One 2007;2:e941

59 Togel F, et al: Administered mesenchymal stem cells protect against ischemic acute renal failure through differentiation-independent mechanisms. Am J Physiol Renal Physiol 2005;289:F31-F42.

60 Ionescu L, et al: Stem cell conditioned medium improves acute lung injury in mice: in vivo evidence for stem cell paracrine action. Am J Physiol Lung Cell Mol Physiol 2012; 303:L967-L977.

$61 \mathrm{Xu} \mathrm{YL}$, et al: Intravenous transplantation of mesenchymal stem cells attenuates oleic acid induced acute lung injury in rats. Chin Med J (Engl) 2012;125:2012-2018.

62 Lee JW, et al: Potential application of mesenchymal stem cells in acute lung injury. Expert Opin Biol Ther 2009;9:1259-1270.

63 Matthay MA: Treatment of acute lung injury: clinical and experimental studies. Proc Am Thorac Soc 2008;5:297-299.

$64 \mathrm{Mei}$ SH, et al: Prevention of LPS-induced acute lung injury in mice by mesenchymal stem cells overexpressing angiopoietin 1 . PLoS Med 2007;4:e269.
65 Stolzing A, et al: Age-related changes in human bone marrow-derived mesenchymal stem cells: consequences for cell therapies. Mech Ageing Dev 2008;129:163-173.

66 Bork S, et al: DNA methylation pattern changes upon long-term culture and aging of human mesenchymal stromal cells. Aging Cell 2010;9:54-63.

67 Roobrouck VD, Ulloa-Montoya F, Verfaillie CM: Self-renewal and differentiation capacity of young and aged stem cells. Exp Cell Res 2008;314:1937-1944.

68 Choumerianou DM, et al: Comparative study of stemness characteristics of mesenchymal cells from bone marrow of children and adults. Cytotherapy 2010;12:881-887.

69 Zhou S, et al: Age-related intrinsic changes in human bone-marrow-derived mesenchymal stem cells and their differentiation to osteoblasts. Aging Cell 2008; 7:335-343.

70 Alt EU, et al: Aging alters tissue resident mesenchymal stem cell properties. Stem Cell Res 2012;8:215-225.

-71 Stolzing A, Bauer E, Scutt A: Suspension cultures of bone-marrow-derived mesenchymal stem cells: effects of donor age and glucose level. Stem Cells Dev 2012;21:2718-2723.

-72 Gorskaya YF, Danilova TA, Nesterenko VG: Age-associated reduction of the count and functional activity of stromal precursor cells can be caused by both true reduction (exhaustion) of cell pool and regulatory effects of the organism. Bull Exp Biol Med 2011;151:210214

73 Mansilla E, et al: Could metabolic syndrome, lipodystrophy, and aging be mesenchymal stem cell exhaustion syndromes? Stem Cells Int 2011;2011:943216.

74 Shao L, et al: Reactive oxygen species and hematopoietic stem cell senescence. Int J Hematol 2011;94:24-32.

75 Kucia M, et al: Reduced number of VSELs in the bone marrow of growth hormone transgenic mice indicates that chronically elevated Igf1 level accelerates age-dependent exhaustion of pluripotent stem cell pool: a novel view on aging. Leukemia 2011;25: 1370-1374.

76 Glauche I, Thielecke L, Roeder I: Cellular aging leads to functional heterogeneity of hematopoietic stem cells: a modeling perspective. Aging Cell 2011;10:457-465.

77 Goldschmidt-Clermont PJ, et al: Inflammation, stem cells and atherosclerosis genetics. Curr Opin Mol Ther 2010;12:712-723.

78 Chilosi M, et al: Epithelial stem cell exhaustion in the pathogenesis of idiopathic pulmonary fibrosis. Sarcoidosis Vasc Diffuse Lung Dis 2010;27:7-18.

79 Durik M, et al: Nucleotide excision DNA repair is associated with age-related vascular dysfunction. Circulation 2012;126:468-478.

80 Gregg SQ, et al: A mouse model of accelerated liver aging caused by a defect in DNA repair. Hepatology 2012;55:609-621. 
81 Gregg SQ, Robinson AR, Niedernhofer LJ: Physiological consequences of defects in ERCC1-XPF DNA repair endonuclease. DNA Repair (Amst) 2011;10:781-791.

82 Goss JR, et al: Premature aging-related peripheral neuropathy in a mouse model of progeria. Mech Ageing Dev 2011;132:437-442.

83 Vo N, et al: Accelerated aging of intervertebral discs in a mouse model of progeria. J Orthop Res 2010;28:1600-1607.

84 Lavasani M, et al: Muscle-derived stem/progenitor cell dysfunction limits healthspan and lifespan in a murine progeria model. Nat Commun 2012;3:608.

85 Conboy IM, et al: Rejuvenation of aged progenitor cells by exposure to a young systemic environment. Nature 2005;433:760-764.

86 Fan M, et al: The effect of age on the efficacy of human mesenchymal stem cell transplantation after a myocardial infarction. Rejuvenation Res 2010;13:429-438.

87 Hermann A, et al: Age-dependent neuroectodermal differentiation capacity of human mesenchymal stromal cells: limitations for autologous cell replacement strategies. Cytotherapy 2010;12:17-30.

88 Guillot PV, et al: Human first-trimester fetal MSC express pluripotency markers and grow faster and have longer telomeres than adult MSC. Stem Cells 2007;25:646-654.

89 Meisel R, et al: Human but not murine multipotent mesenchymal stromal cells exhibit broad-spectrum antimicrobial effector function mediated by indoleamine 2,3-dioxygenase. Leukemia 2011;25:648-654.

90 Krasnodembskaya A, et al: Antibacterial effect of human mesenchymal stem cells is mediated in part from secretion of the antimicrobial peptide LL-37. Stem Cells 2010;28:22292238.

91 Nijnik A, Hancock RE: The roles of cathelicidin LL-37 in immune defences and novel clinical applications. Curr Opin Hematol 2009, 16:41-47.

92 Vallabhaneni KC, Haller H, Dumler I: Vascular smooth muscle cells initiate proliferation of mesenchymal stem cells by mitochondrial transfer via tunneling nanotubes. Stem Cells Dev 2012;21:3104-3113.
93 Gupta N, et al: Intrapulmonary delivery of bone marrow-derived mesenchymal stem cells improves survival and attenuates endotoxin-induced acute lung injury in mice. J Immunol 2007;179:1855-1863.

94 Yamada M, et al: Bone marrow-derived progenitor cells are important for lung repair after lipopolysaccharide-induced lung injury. J Immunol 2004;172:1266-1272.

95 Eggenhofer E, et al: Mesenchymal stem cells are short-lived and do not migrate beyond the lungs after intravenous infusion. Front Immunol 2012;3:297.

-96 Kang SK, et al: Journey of mesenchymal stem cells for homing: strategies to enhance efficacy and safety of stem cell therapy. Stem Cells Int 2012;2012:342968.

$\$ 97$ Geiser T, et al: Pulmonary edema fluid from patients with acute lung injury augments in vitro alveolar epithelial repair by an IL-1beta-dependent mechanism. Am J Respir Crit Care Med 2001;163:1384-1388.

$\checkmark 98$ Ajuebor MN, et al: Role of resident peritoneal macrophages and mast cells in chemokine production and neutrophil migration in acute inflammation: evidence for an inhibitory loop involving endogenous IL-10. J Immunol 1999;162:1685-1691.

99 Takahata Y, et al: Functional expression of beta2 adrenergic receptors responsible for protection against oxidative stress through promotion of glutathione synthesis after Nrf2 upregulation in undifferentiated mesenchymal C3H10T1/2 stem cells. J Cell Physiol 2009;218:268-275.

100 Iyer SS, et al: Cysteine redox potential determines pro-inflammatory IL-1beta levels. PLoS One 2009;4:e5017.

101 Spees JL, et al: Mitochondrial transfer between cells can rescue aerobic respiration. Proc Natl Acad Sci USA 2006;103:12831288.

102 Islam MN, et al: Mitochondrial transfer from bone-marrow-derived stromal cells to pulmonary alveoli protects against acute lung injury. Nat Med 2012;18:759-765.
103 Gupta N, et al: Mesenchymal stem cells enhance survival and bacterial clearance in murine Escherichia coli pneumonia. Thorax 2012;67:533-539.

104 Matthay MA, Wiener-Kronish JP: Intact epithelial barrier function is critical for the resolution of alveolar edema in humans. Am Rev Respir Dis 1990;142:1250-1257.

105 Ware LB, Matthay MA: Alveolar fluid clearance is impaired in the majority of patients with acute lung injury and the acute respiratory distress syndrome. Am J Respir Crit Care Med 2001;163:1376-1383.

$106 \mathrm{Xu}$ J, et al: Mesenchymal stem cell-based angiopoietin-1 gene therapy for acute lung injury induced by lipopolysaccharide in mice. J Pathol 2008;214:472-481.

107 Lee JW, et al: Allogeneic human mesenchymal stem cells for treatment of $E$. coli endotoxin-induced acute lung injury in the ex vivo perfused human lung. Proc Natl Acad Sci USA 2009;106:16357-16362.

108 Ortiz LA, et al: Interleukin 1 receptor antagonist mediates the antiinflammatory and antifibrotic effect of mesenchymal stem cells during lung injury. Proc Natl Acad Sci USA 2007;104:11002-11007.

109 Zhao F, et al: Therapeutic effects of bone marrow-derived mesenchymal stem cells engraftment on bleomycin-induced lung injury in rats. Transplant Proc 2008;40:1700-1705.

110 Lee SH, et al: Modulation of cytokine and nitric oxide by mesenchymal stem cell transfer in lung injury/fibrosis. Respir Res 2010; 11:16.

111 Gonzalez-Rey E, et al: Human adult stem cells derived from adipose tissue protect against experimental colitis and sepsis. Gut 2009;58:929-939.

112 Iyer SS, et al: Effect of bone marrow-derived mesenchymal stem cells on endotoxin-induced oxidation of plasma cysteine and glutathione in mice. Stem Cells Int 2010;2010: 868076.

113 Danchuk S, et al: Human multipotent stromal cells attenuate lipopolysaccharide-induced acute lung injury in mice via secretion of tumor necrosis factor-alpha-induced protein 6. Stem Cell Res Ther 2011;2:27. 